\title{
Actinide chemistry using singlet-paired coupled cluster and its combinations with density functionals
}

\author{
Alejandro J. Garza, ${ }^{1}$ Ana G. Sousa Alencar, ${ }^{1}$ and Gustavo E. Scuseria ${ }^{2}$ \\ ${ }^{1}$ Department of Chemistry, Rice University, Houston, Texas 77251-1892, USA \\ ${ }^{2}$ Department of Chemistry and Department of Physics and Astronomy, Rice University, Houston, \\ Texas 77251-1892, USA
}

(Received 15 October 2015; accepted 4 December 2015; published online 22 December 2015)

\begin{abstract}
Singlet-paired coupled cluster doubles (CCD0) is a simplification of CCD that relinquishes a fraction of dynamic correlation in order to be able to describe static correlation. Combinations of CCD0 with density functionals that recover specifically the dynamic correlation missing in the former have also been developed recently. Here, we assess the accuracy of CCD0 and CCD0+DFT (and variants of these using Brueckner orbitals) as compared to well-established quantum chemical methods for describing ground-state properties of singlet actinide molecules. The $f^{0}$ actinyl series $\left(\mathrm{UO}_{2}^{2+}, \mathrm{NpO}_{2}^{3+}\right.$, $\left.\mathrm{PuO}_{2}^{4+}\right)$, the isoelectronic $\mathrm{NUN}$, and thorium $\left(\mathrm{ThO}, \mathrm{ThO}^{2+}\right)$ and nobelium $\left(\mathrm{NoO}, \mathrm{NoO}_{2}\right)$ oxides are studied. () 2015 AIP Publishing LLC. [http://dx.doi.org/10.1063/1.4938088]
\end{abstract}

\section{INTRODUCTION}

Actinide chemistry represents a challenge for experimental approaches due to the high toxicity and radioactivity of actinide compounds. Accurate computational models are therefore particularly valuable in this area of chemistry. An example of this was the theoretical prediction of $\mathrm{NUO}^{+1}$ and its subsequent discovery by mass spectroscopy. ${ }^{2}$ However, actinide chemistry is also challenging for common quantum chemical approximations: The presence of multiple degenerate, partially filled $f$ orbitals leads to substantial static correlation. Typical techniques for handling static correlation have severe limitations such as lack of size-consistency and size-extensivity and, most restrictively, a combinatorial increase in computational cost with system size. ${ }^{3}$ Furthermore, many of these approaches may miss important dynamic correlation. Other popular methods of quantum chemistry such as Kohn-Sham density functional theory (KS-DFT) or singlereference coupled cluster (CC) are unreliable when static correlation is present ${ }^{4}$ (common CC methods may even diverge or yield complex correlation energies). Recent advances in computational actinide chemistry have been reviewed in Refs. 5 and 6.

Recently, techniques that modify the cluster operator of $\mathrm{CC}$ doubles (CCD) in order to describe static correlation-at the cost of neglecting some dynamic correlation-have been proposed in the literature. These include pair $\mathrm{CCD}^{7-12}$ (pCCD) as well as singlet-paired CCD and Brueckner doubles ${ }^{4,13}$ (CCD0 and BD0, respectively). All of these methods have polynomial scaling and are size-consistent and sizeextensive (provided that the reference determinant be sizeconsistent and size-extensive, which may demand symmetry breaking ${ }^{14}$ ). Furthermore, approaches to incorporate the dynamic correlation absent in CCD0 and BD0 via density functionals (CCD0+DFT) have been developed. ${ }^{13} \mathrm{~A}$ recent study ${ }^{15}$ using pCCD suggests this new type of CC ansätze to be promising for applications in actinide chemistry. Here, we assess the accuracy of CCD0 and CCD0+DFT (and their BD0 variants) as compared to well-established quantum chemical methods for describing ground-state properties of singlet actinide molecules. The $f^{0}$ actinyl series $\left(\mathrm{UO}_{2}^{2+}, \mathrm{NpO}_{2}^{2+}\right.$, $\mathrm{PuO}_{2}^{2+}$ ), the isoelectronic $\mathrm{NUN}$, and thorium $\left(\mathrm{ThO}, \mathrm{ThO}^{2+}\right.$ ) and nobelium $\left(\mathrm{NoO}, \mathrm{NoO}_{2}\right)$ oxides are studied.

\section{THEORY AND METHODS}

\section{A. Singlet-paired coupled cluster doubles (CCD0)}

We give here a minimal description of CCD0; for further details, see Ref. 4. Like standard CC methods, CCD0 uses an exponential wavefunction ${ }^{4}$

$$
\left|\Psi_{\mathrm{CCD} 0}\right\rangle=e^{T_{2}^{[0]}}\left|\Phi_{\mathrm{RHF}}\right\rangle,
$$

where $\left|\Phi_{\mathrm{RHF}}\right\rangle$ is a restricted Hartree-Fock determinant and $T_{2}^{[0]}$ is the cluster operator of singlet-paired double excitations,

$$
T_{2}^{[0]}=\frac{1}{2} \sum_{i j a b} \sigma_{i j}^{a b} P_{a b}^{\dagger} P_{i j}
$$

where $i j$ and $a b$ are indices for occupied and virtual orbitals, respectively, the amplitudes obey the relation $\sigma_{i j}^{a b}=\left(t_{i \uparrow j \downarrow}^{a \uparrow b \downarrow}+t_{i \uparrow j \downarrow}^{b \uparrow a \downarrow}\right) / 2$, and

$$
P_{i j}=\frac{1}{\sqrt{2}}\left(c_{j \uparrow} c_{i \downarrow}+c_{i \uparrow} c_{j \downarrow}\right) .
$$

That is, $P_{a b}$ acting on $\left|\Phi_{\mathrm{RHF}}\right\rangle$ gives a singlet and $e^{T_{2}^{[0]}}$ contains contributions from all singlet-paired excitations. In standard CCD, the cluster operator contains singlet- and triplet-paired contributions, hence capturing more correlation. However, it is the combination of the singlet- and triplet-paired components that cause the failure of $\mathrm{CCD}$ (and coupled cluster singles and doubles (CCSD), singles doubles and triples (CCSDT), etc.) in strongly correlated systems, which results in unphysical correlation energies ${ }^{4}$ (i.e., too large, divergent, or complex energies). Thus, CCD0 relinquishes a fraction of the correlation in exchange for safeguard against this breakdown. 
Singlet-paired BD0 is analogous to CCD0, the only difference being that approximate Brueckner, ${ }^{16-18}$ rather than RHF, orbitals are used as reference,

$$
\left|\Psi_{\mathrm{BD} 0}\right\rangle=e^{T_{2}^{[0]}}\left|\Phi_{\mathrm{BD}}\right\rangle .
$$

Specifically, the approximate Brueckner orbitals in $\left|\Phi_{\mathrm{BD}}\right\rangle$ are those which zero out the amplitudes of single substitutions in a model subspace of single and double substitutions (just like in standard Brueckner doubles).

\section{B. Combination with density functionals (CCD0+DFT)}

The different flavors of CCD0+DFT are discussed in detail in Ref. 13; we just provide here a brief explanation of these techniques for the sake of clarity. There are two categories of CCD0+DFT methods: one that adds parallel spin correlation to CCD0, and another that adds the full contributions from triplet-paired excitations. The first one is derived by noting that the $\mathrm{CCD} 0$ correlation energy is

$$
E_{c}^{\mathrm{CCD} 0}=\sum_{i j a b} \sigma_{i j}^{a b} v_{i \uparrow j \downarrow}^{a \uparrow b \downarrow},
$$

where $v_{i j}^{a b}=\langle i j \mid a b\rangle$ is a two-electron integral in the Dirac notation. Hence, there are no contributions to the correlation from pairs of same-spin electrons. One can thus add (without double counting) the equal spin correlation to CCD0 using a density functional approximation (DFA). For a singlet, the correlation energy would be

$$
E_{c}^{\mathrm{CCD} 0+\mathrm{pDFT}}=E_{c}^{\mathrm{CCD} 0}+2 E_{c \uparrow \uparrow}^{\mathrm{DFA}}\left[n_{\uparrow}, n_{\downarrow}\right],
$$

where the "p" in pDFT is for "parallel-spin" and $E_{c \alpha \alpha}^{\mathrm{DFA}}$ is the DFA correlation for the spin- $\alpha$ density.

The second category of CCD0+DFT is derived by noting that the full double excitations cluster operator $T_{2}$ used in the latter can be expressed as

$$
T_{2}=T_{2}^{[0]}+T_{2}^{[1]},
$$

where $T_{2}^{[0]}$ is defined above and $T_{2}^{[1]}$ is the triplet-paired component of $T_{2}$,

$$
T_{2}^{[1]}=\frac{1}{2} \sum_{i j a b} \pi_{i j}^{a b} \mathbf{Q}_{\mathbf{a b}}^{\dagger} \cdot \mathbf{Q}_{\mathbf{i j}},
$$

where $\mathbf{Q}_{\mathrm{ij}}$ is a vector $\mathbf{Q}_{\mathbf{i j}}=\left(Q_{i j}^{+}, Q_{i j}^{0}, Q_{i j}^{-}\right)^{t}$ whose components are

$$
\begin{aligned}
Q_{i j}^{+} & =c_{j \uparrow} c_{i \uparrow}, \quad Q_{i j}^{-}=c_{j \downarrow} c_{i \downarrow}, \\
Q_{i j}^{0} & =\frac{1}{\sqrt{2}}\left(c_{j \uparrow} c_{i \downarrow}-c_{i \uparrow} c_{j \downarrow}\right) \\
& =\frac{1}{\sqrt{2}}\left(c_{j \uparrow} c_{i \downarrow}+c_{j \downarrow} c_{i \uparrow}\right) .
\end{aligned}
$$

Thus, CCD0 misses not only parallel spin correlation, but also the $m=0$ channel of $T_{2}^{11}$. For a closed shell, the $m=+1,0$, and -1 components of $T_{2}^{[1]}$ contribute equally to the correlation. We may therefore incorporate the opposite spin correlation missing in $\mathrm{CCD} 0+\mathrm{pDFT}$ by adding $E_{c}^{\mathrm{DFA}}\left[n_{\uparrow}, 0\right]$ once more to the (closed-shell) energy

$$
E_{c}^{\mathrm{CCD} 0+\mathrm{tDFT}}=E_{c}^{\mathrm{CCD} 0}+3 E_{c \uparrow \uparrow}^{\mathrm{DFA}}\left[n_{\uparrow}, n_{\downarrow}\right],
$$

where the " $\mathrm{t}$ " in tDFT indicates that the full contributions from the triplet-paired component of $T_{2}$ are being taken into account.

In the interest of simplicity and intuitiveness, the derivation of CCD0+DFT given here is somewhat heuristic. However, as shown in the Appendix of Ref. 13, it is also possible to derive CCD0+DFT formally using Levy-Liebtype $^{19,20}$ constrained search functionals. The basic idea is that one can divide the electron-electron interaction in complementary singlet- and triplet-pairing components. There is then a wavefunction that solves the Hamiltonian with the singlet-pairing interaction only and a complementary density functional for triplet-pairing interaction. This functional can be written in terms of the parallel spin correlation of the usual Levy-Lieb universal functional, plus a term corresponding to the $m=0$ part of the triplet-pairing contribution to the energy. If one neglects this last term, a method analogous to $\mathrm{CCD} 0+\mathrm{pDFT}$ is obtained; if it is approximated to be equal to half the contribution of the parallel spins (which is reasonable due to the symmetry of the triplet-pairing interaction), a method analogous to CCD0+tDFT is obtained. The additional assumptions done in CCD0+DFT are that the wavefunction that solves for the singlet-pairing interacting Hamiltonian is well approximated by $\mathrm{CCD} 0$, that the density of the reference determinant is accurate, and that the parallel spin correlation of the approximate meta-generalized gradient approximations (GGAs) is also accurate. For details, the reader is referred to the Appendix of Ref. 13; the main purpose of commenting on this here is to point out that CCD0+DFT can be considered as a rigorous way to incorporate dynamic correlation in CCD0 while avoiding double counting.

\section{Parallel spin functionals for CCDO+DFT}

The CCD0+DFT methods described above require a spin resolution for DFA correlation in order to approximate $E_{c \uparrow \uparrow}^{\mathrm{DFA}}\left[n_{\uparrow}, n_{\downarrow}\right]$. For completeness, we discuss this topic briefly here. The simplest spin resolution for the DFA correlation is the exchange-like ansatz of Stoll et al. ${ }^{21}$

$$
E_{c \uparrow \uparrow}^{\mathrm{DFA}}\left[n_{\uparrow}, n_{\downarrow}\right]=E_{c}^{\mathrm{DFA}}\left[n_{\uparrow}, 0\right] .
$$

This equation can be used for the local density approximation (LDA), GGAs, and meta-GGAs that are rooted on the homogeneous electron gas. (Not all DFAs have a meaningful spin resolution. The Lee-Yang-Parr ${ }^{22}$ functional, for example, models all correlations as being opposite spin.) The use of meta-GGAs is most desirable because these functionals are free of one-electron self-interaction, and thus, BD0+DFT with a meta-GGA is exact for two-electron singlets using the spin resolution of Eq. (12). Here, we use two nonempirical meta-GGAs in combination with CCD0 and BD0: the Tao-Perdew-Staroverov-Scuseria ${ }^{23}$ (TPSS) functional and the strongly constrained and appropriately normed (SCAN) functional of Sun $e t$ al. ${ }^{24}$

Equation (12) is an educated guess which is exact only for fully spin-polarized densities and in the high-density limit of the uniform electron gas. ${ }^{25}$ In the case of SCAN, however, it is possible to compose an improved guess for the same-spin correlation..$^{13}$ This is because SCAN constructs the correlation 
TABLE I. Summary of CC0+DFT methods employed here. The notation and closed-shell energy formulas are given; $\mathrm{CC} 0$ can refer to $\mathrm{CCD} 0$ or BD0 and the densities are from the RHF or Brueckner reference determinants, respectively; the " $\mathrm{p}$ " in pDFT is for parallel spin; the " $\mathrm{t}$ " in tDFT is for triplet-pairing component; DFT may refer to TPSS or SCAN; and $E_{c \uparrow \uparrow}^{\mathrm{rSCAN}}$ is the spin-up SCAN correlation using the spin resolution of Section II C.

\begin{tabular}{cl}
\hline \hline Method & \multicolumn{1}{c}{ Energy formula } \\
\hline $\mathrm{CC} 0+\mathrm{pDFT}$ & $E^{\mathrm{CC} 0}+2 E_{c}^{\mathrm{DFA}}\left[n_{\uparrow}, 0\right]$ \\
$\mathrm{CC} 0+\mathrm{tDFT}$ & $E^{\mathrm{CC} 0}+3 E_{c}^{\mathrm{DFA}}\left[n_{\uparrow}, 0\right]$ \\
$\mathrm{CC} 0+\mathrm{prSCAN}$ & $E^{\mathrm{CC} 0}+2 E_{c \uparrow \uparrow}^{\mathrm{rSCAN}}\left[n_{\uparrow}, n_{\downarrow}\right]$ \\
$\mathrm{CC} 0+\operatorname{trSCAN}$ & $E^{\mathrm{CC} 0}+3 E_{c \uparrow \uparrow}^{\mathrm{rSCAN}}\left[n_{\uparrow}, n_{\downarrow}\right]$ \\
\hline \hline
\end{tabular}

energy density $\varepsilon_{c}$ as ${ }^{24}$

$$
\varepsilon_{c}=\varepsilon_{c}^{1}+f_{c}(\alpha)\left[\varepsilon_{c}^{0}-\varepsilon_{c}^{1}\right],
$$

where $f_{c}(\alpha)$ is a function that depends on the kinetic energy density (see the supplementary material of Ref. 24), and $\varepsilon_{c}^{\alpha=0}$ and $\varepsilon_{c}^{\alpha=1}$ are the single orbital and uniform density limits, respectively, for the correlation energy density. It is thus logical to write the spin-up correlation energy density for SCAN as

$$
\varepsilon_{c}^{\uparrow \uparrow}=\varepsilon_{c}^{1 \uparrow \uparrow}+f_{c}(\alpha)\left[\varepsilon_{c}^{0 \uparrow \uparrow}-\varepsilon_{c}^{1 \uparrow \uparrow}\right] .
$$

Furthermore, $\varepsilon_{c}^{0 \uparrow \uparrow}=0$ because there is no parallel-spin correlation for two electrons in the same spatial orbital. Hence,

$$
\varepsilon_{c}^{\uparrow \uparrow}=\varepsilon_{c}^{1 \uparrow \uparrow}-f_{c}(\alpha) \varepsilon_{c}^{1 \uparrow \uparrow}
$$

so that $\varepsilon_{c}^{\uparrow \uparrow}$ depends only on the uniform density limit of the spin-up correlation energy density, $\varepsilon_{c}^{1 \uparrow \uparrow}$. The spin resolution of $\varepsilon_{c}$ in the uniform electron gas has been parametrized by Gori-Giorgi and Perdew ${ }^{25}$ in terms for fractions $F_{\sigma \sigma^{\prime}}$ such that $\varepsilon_{c}^{\sigma \sigma^{\prime}}=\varepsilon_{c} F_{\sigma \sigma^{\prime}}$. Thus, we compute $\varepsilon_{c}^{1 \uparrow}=\varepsilon_{c} F_{\uparrow \uparrow}$ using the expression for $F_{\uparrow \uparrow}$ given in Equation (9) of Ref. 25. We term the variation of SCAN using this spin resolution as "rSCAN." For convenience of the reader, Table I summarizes the CCD0+DFT methods described and employed in this work. Further details regarding CCD0+DFT, including discussion about the spin resolutions, may be consulted in Ref. 13.

\section{Computational details}

All calculations were carried out using a development version of GaUssian ${ }^{26}$ in which the CCD0 and CCD0+DFT methods have been implemented. As in Ref. 13, CCD0+DFT calculations are done in a non-self-consistent manner: the DFA correlation is evaluated in a single-shot, post-CCD0 calculation with the densities from the reference determinant. CCD0+DFT geometry optimizations and harmonic vibrational frequencies were computed numerically using a convergence threshold of $1 \times 10^{-9}$ hartree on the CCD0 energy and the largest of the preset grids in Gaussian for integrating the density functional (Integral=SuperFine keyword). CCD0 geometries and frequencies were evaluated analytically, and we verified that the numerical procedure for determining these properties with CCD0+DFT agreed with analytical results for CCD0. These same specifications were followed in BD0 and BD0+DFT calculations. Unless otherwise indicated, all calculations employ the Stuttgart relativistic small-core effective core potential ${ }^{27-29}$ (RSC ECP) basis for actinide atoms and the aug-cc-pVDZ basis for the light atoms. These basis sets have been shown to be adequate for the type of calculations carried out here. ${ }^{30}$ Spin-orbit coupling effects are neglected as they are not important for the closed-shell species studied in this work. ${ }^{6,31}$ The results reported here are all in gas phase media.

\section{RESULTS AND DISCUSSION}

\section{A. Uranyl cation $\left(\mathrm{UO}_{2}^{2+}\right)$}

The uranyl ion $\mathrm{UO}_{2}^{2+}$ is considered the most important of the actinyls due to its ubiquity: In practical applications, nuclear reactors usually rely on uranium to fuel nuclear chain reactions, and $\mathrm{UO}_{2}^{2+}$ is the most common form of uranium in aqueous solution. $\mathrm{UO}_{2}^{2+}$ is highly toxic and its study is motivated by the need for knowledge regarding soluble actinide complexes, which are important for nuclear waste disposal and environmental transport. ${ }^{6}$ The "bare" $\mathrm{UO}_{2}^{2+}$ ion has also been observed experimentally via mass-spectrometric techniques. ${ }^{32}$ The uranyl cation has therefore been studied extensively. ${ }^{6,30,31,33-35}$ Here, we study this species in the gas phase due to the availability of data from high-level calculations to compare with, as the accuracy of CCD0 and CCD0+DFT for actinide compounds has not yet been established. To the best of out knowledge, there is no experimental data for the properties of $\mathrm{UO}_{2}^{2+}$ here calculated.

Table II compiles predictions by various methods for the bond lengths $\left(R_{e}\right)$ and harmonic vibrational frequencies $\left(\omega_{e}\right)$ of $\mathrm{UO}_{2}^{2+}$. Some of these data have been taken from the literature and not all calculations use the same basis set; however, the results should be roughly comparable because the bases are all of similar, good quality. The highest level methods in this table are $\operatorname{CCSD}(\mathrm{T})$ and CASPT2(12,12); $\mathrm{CCSD}(\mathrm{T})$ results are considered to be reliable for uranyl. ${ }^{6,33,34}$ CCD0 is in good agreement with $\operatorname{CCSD}(\mathrm{T})$ except for a large overestimation of the bending frequency $\omega_{\beta}$. This problem persists in CCD0+pTPSS but is alleviated by combinations of CCD0 with SCAN. Compared to CCD0, BD0 provides a much better estimate of $\omega_{\beta}$. Whereas BD0+pTPSS tends to give a too short $R_{e}$ and too large frequencies, $\mathrm{BD} 0+\mathrm{SCAN}$ methods are, overall, in excellent agreement with $\operatorname{CCSD}(\mathrm{T})$. It is worth noting that the trends for the $\mathrm{UO}_{2}^{2+}$ frequencies are the same as those observed for a set of ten first- and second-row diatomics in Ref. 13: CCD0+pTPSS overestimates the frequencies, while CCD0+SCAN combination improves results. The trend is similar for BD0+DFT methods, which are more accurate than their CCD0+DFT counterparts.

CCD0, BD0, and their combinations with DFT (in particular those with SCAN) fare well against other methods. Results from HF and KS-DFT methods in Table II suggest that the description of $\mathrm{UO}_{2}^{2+}$ is dependent on the amount of $\mathrm{HF}$ exchange in the functional: more HF exchange leads to shorter bond lengths and higher frequencies. This dependence can make common hybrids unreliable for high accuracy work. The "cousin" of CCD0, pCCD, underestimates $R_{e}$ more than all 
TABLE II. Bond lengths $\left(R_{e}\right.$ in $\AA$ ) and harmonic vibrational frequencies $\left(\omega_{e}\right.$ in $\left.\mathrm{cm}^{-1}\right)$ for $\mathrm{UO}_{2}^{2+}\left(D_{\infty h}\right)$ calculated by various methods. Results computed in this work appear in the top part of the table; results compiled from the literature at the bottom. To the best of our knowledge, no experimental data are available for this species.

\begin{tabular}{|c|c|c|c|c|}
\hline $\begin{array}{l}\text { This work } \\
\text { method }\end{array}$ & $R_{e}$ & $\omega_{\text {as }}$ & $\omega_{\mathrm{s}}$ & $\omega_{\beta}$ \\
\hline CCD0 & 1.698 & 1129 & 1053 & 541 \\
\hline CCD0+pTPSS & 1.681 & 1162 & 1091 & 508 \\
\hline $\mathrm{CCD} 0+\mathrm{pSCAN}$ & 1.686 & 1143 & 1073 & 230 \\
\hline $\mathrm{CCD} 0+$ prSCAN & 1.687 & 1148 & 1078 & 223 \\
\hline $\mathrm{CCD} 0+\operatorname{trSCAN}$ & 1.683 & 1157 & 1086 & 226 \\
\hline BD0 & 1.700 & 1143 & 1049 & 253 \\
\hline BD0+pTPSS & 1.685 & 1130 & 1061 & 379 \\
\hline $\mathrm{BD} 0+\mathrm{pSCAN}$ & 1.694 & 1112 & 1044 & 226 \\
\hline $\mathrm{BD} 0+$ prSCAN & 1.693 & 1121 & 1052 & 220 \\
\hline $\mathrm{BD} 0+\operatorname{trSCAN}$ & 1.689 & 1126 & 1057 & 381 \\
\hline CCSD & 1.696 & 1151 & 1059 & 202 \\
\hline $\operatorname{CCSD}(\mathrm{T})$ & 1.702 & 1113 & 1025 & 192 \\
\hline $\mathrm{HF}$ & 1.648 & 1293 & 1220 & 267 \\
\hline PBE & 1.715 & 1086 & 985 & 123 \\
\hline PBEh & 1.684 & 1175 & 1082 & 187 \\
\hline LC- $\omega \mathrm{PBE}$ & 1.674 & 1213 & 1125 & 192 \\
\hline $\begin{array}{l}\text { Literature } \\
\text { method }\end{array}$ & $R_{e}$ & $\omega_{\mathrm{as}}$ & $\omega_{\mathrm{s}}$ & $\omega_{\beta}$ \\
\hline $\mathrm{pCCD}^{\mathrm{a}}$ & 1.669 & $\ldots$ & 1060 & $\ldots$ \\
\hline $\operatorname{CCSD}(\mathrm{T})^{\mathrm{b}}$ & 1.690 & 1120 & 1035 & 178 \\
\hline $\mathrm{MP} 2^{\mathrm{a}}$ & 1.745 & $\ldots$ & 854 & $\ldots$ \\
\hline $\mathrm{MP} 2^{\mathrm{c}}$ & 1.724 & 1052 & 941 & 277 \\
\hline $\operatorname{CASSCF}(10,10)^{\mathrm{a}}$ & 1.694 & $\ldots$ & 1085 & $\ldots$ \\
\hline $\operatorname{CASSCF}(12,12)^{\mathrm{a}}$ & 1.707 & $\ldots$ & 1034 & $\ldots$ \\
\hline CAS-srPBE $(12,12)^{\mathrm{d}}$ & 1.684 & $\ldots$ & $\ldots$ & $\ldots$ \\
\hline CAS-srLDA $(12,12)^{\mathrm{d}}$ & 1.684 & $\ldots$ & $\ldots$ & $\ldots$ \\
\hline $\operatorname{CASPT} 2(12,12)^{\mathrm{e}}$ & 1.714 & 1153 & 1043 & $\ldots$ \\
\hline CASPT2 $(12,12)^{\mathrm{f}}$ & 1.705 & 1066 & 959 & $\ldots$ \\
\hline
\end{tabular}

${ }^{\mathrm{a}}$ From Ref. 15 using the cc-pVDZ basis on O.

${ }^{\mathrm{b}}$ From Ref. 33, RSC $+3 g$ on $\mathrm{U}$ and aug-cc-pVQZ on $\mathrm{O}$.

${ }^{\mathrm{c}}$ From Ref. 34, RSC $+2 g$ on U and aug-cc-pVDZ on O.

${ }^{\mathrm{d}}$ From Ref. 31, RECP $(14 s 13 p 10 d 8 f 6 g) /[6 s 6 p 5 d 4 f 3 g]$ on $\mathrm{U}$ and $(4 s 5 p 1 d) /$ $[2 s 3 p 1 d]$ on $\mathrm{O}$.

${ }^{\mathrm{e}}$ From Ref. 35, RSC on U and $4 s 3 p 2 d$ ANO-S on O

${ }^{\mathrm{f}}$ From Ref. 35, RSC on U and $4 s 3 p 2 d 1 f$ ANO-L on O.

other methods except HF. In pCCD, a singlet pairing scheme is also employed, but only the diagonal (optimized) terms are retained. A better performance of $\mathrm{CCD} 0$ as compared to pCCD could be expected because the former contains more contributions in the cluster operator: the $T$ operator of $\mathrm{pCCD}$ is

$$
T_{\mathrm{pCCD}}=\sum_{i a} t_{i}^{a} c_{a \uparrow}^{\dagger} \uparrow_{a \downarrow}^{\dagger} c_{i \downarrow} c_{i \uparrow},
$$

which is only a part of the $T^{[0]}$ of CCD0. Although pCCD normally compensates for the missing terms via an orbital optimization, this optimization is nontrivial and can have multiple solutions. Nonetheless, pCCD has the advantage of having lower scaling. The cost of CCD0 is determined by the cost of solving the CCD0 equations with symmetrized amplitudes. Thus, the scaling of CCD0 is the same as that of $\mathrm{CCD}, O\left(N^{6}\right)$. For pCCD, the cost of solving the pertinent CC equations is a remarkably low $O\left(N^{3}\right)$, although a $O\left(N^{5}\right)$ basis transformation is required for the orbital optimization (and this is important for achieving good results and ensuring size consistency). Compared to the traditional, "gold-standard" $\operatorname{CCSD}(\mathrm{T}), \mathrm{CCD} 0$, and CCD0+DFT are an order of magnitude lower in cost, while providing similar results and being more reliable for static correlation. ${ }^{4}$

Table II also shows CAS-srDFT results from Ref. 31. These methods belong to a class of techniques that complement long-range wavefunction two-body energy with short-range DFT Hartree-exchange-correlation. The idea is to capture the dynamic correlation, which is short-range, with DFT and avoid double counting by range separation; an approach to avoid double counting that is very different from that used in CCD0+DFT. The CAS-srDFT bond lengths are comparable to those of CCD0+DFT, although the latter has the advantage that it does not neglect effects of static correlation in the short-range. Complete active space self-consistent field (CASSCF) and CASPT2 (complete active space second order perturbation theory) results are also comparable to those from CCD0/BD0 (and their +DFT combinations), whereas MP2 gives too large bond lengths.

\section{B. Neptunyl and plutonyl $\left(\mathrm{NpO}_{2}^{3+}\right.$ and $\left.\mathrm{PuO}_{2}^{4+}\right)$}

The increased charge in neptunyl and plutonyl $\left(\mathrm{NpO}_{2}^{3+}\right.$ and $\mathrm{PuO}_{2}^{4+}$ ) as compared to the isoelectronic uranyl enhances degeneracies, bolstering static correlation. This makes these ions more challenging to describe than $\mathrm{UO}_{2}^{2+}$. In fact, $\operatorname{CCSD}(\mathrm{T})$ results reported in the literature for these systems come with a warning: The $T_{1}$ diagnostics for neptunyl and plutonyl are 0.22 and 0.35 , respectively. ${ }^{34}$ Empirically, $\operatorname{CCSD}(\mathrm{T})$ predictions are considered unreliable when $T_{1}>0.2^{36}$ (although the norm of $T_{2}$ is probably a more reliable and better indicator of static correlation, ${ }^{4}$ we did not find these data in the literature).

Table III shows $R_{e}$ and $\omega_{e}$ results for $\mathrm{NpO}_{2}^{3+}$. The methods for which results are shown in this table all predict a linear geometry for neptunyl. The prediction of a linear geometry can be considered a success for CCD0+DFT methods because it has been shown that a correct description of both exchange and correlation is necessary for this. ${ }^{31}$ Other MR+DFT that avoids double counting typically does so at the cost of introducing some (inexact) DFT exchange. Popular KS-DFT functionals like LDA, PBE, and B3LYP predict bent geometries for neptunyl. ${ }^{31}$ As a result, MR+DFT combinations having substantial DFT exchange also tend toward bent geometries. ${ }^{31}$ We also note that $\operatorname{CCSD}(\mathrm{T})$ gives results comparable to CASSCF, BD0, and BD0+DFT. Considering this and the fact that the $T_{1}$ diagnostic of $\operatorname{CCSD}(\mathrm{T})^{34}\left(T_{1}=0.22\right)$ is close to the limit of what is considered reliable ${ }^{36}(0.20)$, it seems like the $\operatorname{CCSD}(\mathrm{T})$ results are salvageable for $\mathrm{NpO}_{2}^{3+}$. MP2 is not reliable for the $f^{0}$ actinyl series isoelectronic to uranyl beyond uranyl itself; the bond lengths in neptunyl appear to be largely overestimated, while the frequencies are underestimated.

In the case of $\mathrm{PuO}_{2}^{4+}$, the $\mathrm{CCD} 0$ and $\mathrm{BD} 0$ geometry optimizations resulted in linear structures but with imaginary bending frequencies of 149 and $227 \mathrm{~cm}^{-1}$, respectively. Attempts to optimize the structure starting from a bond angle of about $160^{\circ}$ (a minimum at a fixed bond length) resulted 
TABLE III. Bond lengths $\left(R_{e}\right.$ in $\AA$ ) and harmonic vibrational frequencies $\left(\omega_{e}\right.$ in $\left.\mathrm{cm}^{-1}\right)$ for the neptunyl ion $\mathrm{NpO}_{2}^{3+}\left(D_{\infty h}\right)$ calculated by various methods. To the best of our knowledge, no experimental data are available for this species.

\begin{tabular}{lcccc}
\hline \hline $\begin{array}{l}\text { This work } \\
\text { method }\end{array}$ & $R_{e}$ & $\omega_{\text {as }}$ & $\omega_{\mathrm{s}}$ & $\omega_{\beta}$ \\
\hline CCD0 & 1.662 & 1183 & 1076 & 217 \\
CCD0+pTPSS & 1.645 & 1188 & 1121 & 221 \\
CCD0+pSCAN & 1.654 & 1157 & 1092 & 216 \\
CCD0+prSCAN & 1.656 & 1150 & 1085 & 216 \\
CCD0+trSCAN & 1.652 & 1161 & 1095 & 215 \\
BD0 & 1.671 & 1142 & 1021 & 205 \\
BD0+pTPSS & 1.653 & 1135 & 1071 & 201 \\
BD0+pSCAN & 1.664 & 1095 & 1033 & 190 \\
BD0+prSCAN & 1.665 & 1091 & 1030 & 191 \\
BD0+trSCAN & 1.663 & 1095 & 1033 & 191 \\
\hline Literature & & & & \\
method & $R_{e}$ & $\omega_{\text {as }}$ & $\omega_{\mathrm{s}}$ & $\omega_{\beta}$ \\
\hline${\text { CCSD }(T)^{\mathrm{a}}}_{\text {MP2 }}$ & 1.682 & 1106 & 990 & 141 \\
${\text { CASSCF }(12,12)^{\mathrm{a}}}_{\text {CASSCF }(12,16)^{\mathrm{a}}}$ & 1.757 & 900 & 879 & 106 \\
\hline \hline
\end{tabular}

${ }^{\mathrm{a}}$ From Ref. $34, \mathrm{RSC}+2 g$ on Np and aug-cc-pVDZ on O.

in the geometry going back to linear with an extended bond length without achieving convergence after many iterations. Straka et al. ${ }^{34}$ reported that $\mathrm{CAS}(12,16)$ predicts the $\mathrm{PuO}_{2}^{4+}$ system to disintegrate and that $\operatorname{CCSD}(\mathrm{T})$ results are highly unreliable $\left(T_{1}=0.35\right)$. Plutonium has four common oxidation states $\mathrm{Pu}(\mathrm{III}), \mathrm{Pu}(\mathrm{Iv}), \mathrm{Pu}(\mathrm{v})$, and $\mathrm{Pu}(\mathrm{vI})$; the highest known oxidation state of Pu in aqueous solution is VII and is only stable in strong alkaline medium. However, the formal oxidation state of plutonium in $\mathrm{PuO}_{2}^{4+}$ is vIII. The too-high charge of $\mathrm{Pu}$ in $\mathrm{PuO}_{2}^{4+}$ brings the actinide's low-lying $f$ orbitals closer to the atom and makes them less suitable for bonding. Considering all this, it seems very likely that $\mathrm{PuO}_{2}^{4+}$ is not a stable gas phase species. Nevertheless, Tsushima ${ }^{37}$ has theorized a possible synthesis for $\mathrm{PuO}_{5} \mathrm{OH}^{3-}$ in strong alkaline solution, and Huang et al. ${ }^{38}$ have recently reported theoretical evidence for the stability of a $\mathrm{PuO}_{2} \mathrm{~F}_{4}$ complex.

\section{Uranium dinitride (NUN)}

The linear NUN molecule has been studied theoretically and observed experimentally. ${ }^{35,39-42}$ The interest in this compound stems mostly from its similarity to the important uranyl ion to which it is isoelectronic. Results for $R_{e}$ and $\omega_{e}$ computed by various methods here and in previous works are listed in Table IV. General trends are similar to those observed for uranyl: CCD0 and CCD0+DFT methods are in good agreement with available CASPT2 and $\operatorname{CCSD}(\mathrm{T})$ methods, although the CASPT2 vibrational frequencies are somewhat lower than those predicted by coupled cluster methods. Results by KS-DFT methods depend on the amount of exchange, with the bond length being reduced as more exchange is incorporated. In addition, PBEh and LC- $\omega$ PBE yield a linear geometry but $\mathrm{PBE}$ shows an imaginary bending frequency
TABLE IV. Bond lengths $\left(\boldsymbol{R}_{\boldsymbol{e}}\right.$ in $\AA$ ) and harmonic vibrational frequencies $\left(\omega_{e}\right.$ in $\left.\mathrm{cm}^{-1}\right)$ for the NUN molecule $\left(D_{\infty h}\right)$ calculated by various methods.

\begin{tabular}{lcccc}
\hline \hline $\begin{array}{l}\text { This work } \\
\text { method }\end{array}$ & $R_{e}$ & $\omega_{\text {as }}$ & $\omega_{\mathrm{s}}$ & $\omega_{\beta}$ \\
\hline CCD0 & 1.735 & 1098 & 1059 & 188 \\
CCD0+pTPSS & 1.717 & 1165 & 1102 & 119 \\
CCD0+pSCAN & 1.724 & 1140 & 1079 & 198 \\
CCD0+prSCAN & 1.724 & 1151 & 1089 & 114 \\
CCD0+trSCAN & 1.719 & 1162 & 1100 & 117 \\
BD0 & 1.736 & 1109 & 1067 & 239 \\
BD0+pTPSS & 1.721 & 1142 & 1080 & 109 \\
BD0+pSCAN & 1.730 & 1111 & 1051 & 205 \\
BD0+prSCAN & 1.729 & 1129 & 1067 & 113 \\
BD0+trSCAN & 1.725 & 1136 & 1074 & 118 \\
CCSD & 1.733 & 1119 & 1050 & 155 \\
PBE & 1.738 & 1085 & 1032 & $37 i$ \\
PBEh & 1.713 & 1154 & 1113 & 118 \\
LC- $\omega$ PBE $^{2}$ & 1.701 & 1200 & 1166 & 129 \\
\hline Literature $_{\text {method }}$ & & & & \\
\hline HF $^{\mathrm{a}}$ & $R_{e}$ & $\omega_{\text {as }}$ & $\omega_{\mathrm{s}}$ & $\omega_{\beta}$ \\
CCSD(T) $^{\mathrm{a}}$ & 1.640 & $\ldots$ & $\ldots$ & $\ldots$ \\
MP2 $^{\mathrm{a}}$ & 1.722 & $\ldots$ & $\ldots$ & $\ldots$ \\
CAS-srLDA & 1.721 & $\ldots$ & $\ldots$ & $\ldots$ \\
CAS-srPBE $^{\mathrm{a}}$ & 1.710 & $\ldots$ & $\ldots$ & $\ldots$ \\
CASPT2(12,12) $^{\mathrm{b}}$ & 1.710 & $\ldots$ & $\ldots$ & $\ldots$ \\
Expt. $^{\mathrm{c}}$ & 1.735 & 1031 & 969 & $\ldots$ \\
Expt. $^{\mathrm{d}}$ & $\ldots$ & 1077 & $\ldots$ & $\ldots$ \\
Expt. $^{\mathrm{e}}$ & $\ldots$ & 1051 & 1008 & $\ldots$ \\
\hline \hline & $\ldots$ & 1051 & $\ldots$ & $\ldots$ \\
\hline
\end{tabular}

\begin{tabular}{llllllll}
\hline${ }^{a}$ From & Ref. & 31, & RECP & $(14 s 13 p 10 d 8 f 6 g) /[6 s 6 p 5 d 4 f 3 g]$ & on & $\mathrm{U}$ & and
\end{tabular} $(4 s 5 p 1 d) /[2 s 3 p 1 d]$ on $\mathrm{O}$.

${ }^{\mathrm{b}}$ From Ref. 35 , RSC on U and $4 s 3 p 2 d 1 f$ ANO-L on O

${ }^{\mathrm{c}}$ From Ref. 39.

${ }^{\mathrm{d}}$ From Ref. 40

${ }^{\mathrm{e}}$ From Ref. 41.

for the linear structure. Again, HF gives bond lengths that are too-short, although MP2 appears to give more reasonable bond lengths for this species.

\section{Thorium oxides $\mathrm{ThO}$ and $\mathrm{ThO}^{2+}$}

$\mathrm{ThO}$ is the most studied (experimentally and theoretically) of the actinide monoxides. ${ }^{6} \mathrm{ThO}^{2+}$ is also of interest because $\mathrm{Th}(\mathrm{IV})$ is the most common oxidation state for thorium. The bonding in $\mathrm{ThO}^{2+}$ is furthermore peculiar: it forms a triple bond and leaves the lone-pair orbital on the thorium empty (see Fig. 1), as occurs in the "isovalent" TiC. ${ }^{43}$ Interest in thorium chemistry arises mainly from potential applications of the thorium fuel cycle - the transmutation of the abundant ${ }^{232}$ Th into artificial ${ }^{233} \mathrm{U}$, which is the actual fuel in the nuclear chain reaction. In fact, thorium has been touted as potential "wonder fuel" 44 due to certain advantages over uranium like greater abundance and better resistance to nuclear weapons proliferation, albeit the latter advantage has been contended. ${ }^{44}$

Table V compares the $R_{e}$ and $\omega_{\mathrm{e}}$ for $\mathrm{ThO}$ and $\mathrm{ThO}^{2+}$ obtained by various methods. The data from CCD0, BD0, and their combinations with DFT are all very close to available CASPT2 and experimental data from the literature. Once 


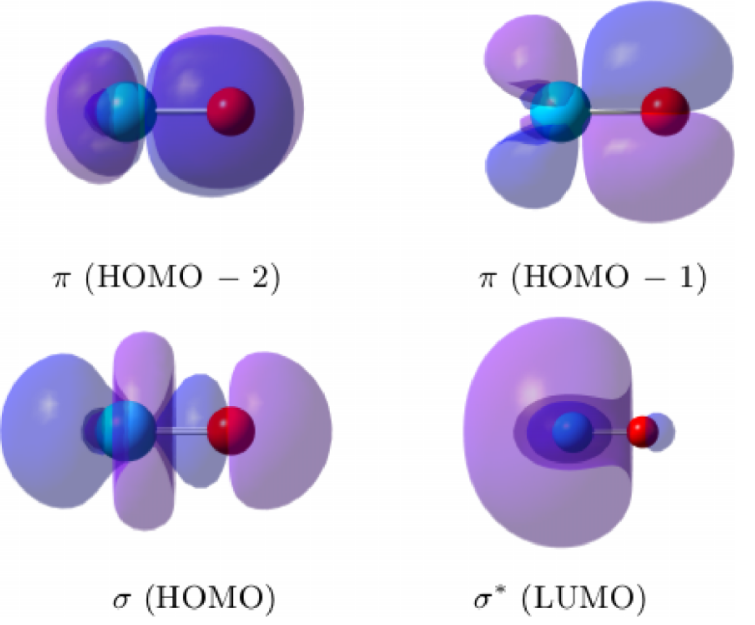

FIG. 1. The highest three occupied and lowest unoccupied (Hartree-Fock) molecular orbitals of $\mathrm{ThO}^{2+}$.

more, combinations using the SCAN functional tend to be closer to the reference CASPT2 values, though all the combinations presented here provide satisfactory results. The trend of DFT methods to shorten the bond length as more Fock exchange is incorporated that was observed in the previous cases is also present here.

\section{E. Nobelium oxides $\mathrm{NoO}$ and $\mathrm{NoO}_{2}$}

The chemistry of nobelium is largely uncharacterized, which offers a possibility for theoretical methods to provide unique insight into it. Table VI shows bond lengths and harmonic frequencies for $\mathrm{NoO}$ and $\mathrm{NoO}_{2}$ computed by $\mathrm{BD} 0$, BD0+DFT, and some standard coupled cluster and KS-DFT methods. The $\omega_{e}$ for NoO is omitted because the floppy (long and rather weak) bond of this molecule leads to somewhat

TABLE V. Bond lengths $\left(\boldsymbol{R}_{\boldsymbol{e}}\right.$ in $\AA$ ) and harmonic vibrational frequencies $\left(\omega_{e}\right.$ in $\left.\mathrm{cm}^{-1}\right)$ for $\mathrm{ThO}$ and $\mathrm{ThO}^{2+}$ calculated by various methods.

\begin{tabular}{lccccc}
\hline \hline & \multicolumn{2}{c}{ ThO } & & \multicolumn{2}{c}{ ThO $^{2+}$} \\
\cline { 2 - 3 } \cline { 5 - 6 } Method & $R_{e}$ & $\omega_{\mathrm{e}}$ & & $R_{e}$ & $\omega_{\mathrm{e}}$ \\
\hline CCD0 & 1.869 & 880 & 1.792 & 1000 \\
CCD0+pTPSS & 1.847 & 906 & 1.774 & 1030 \\
CCD0+pSCAN & 1.856 & 890 & 1.781 & 979 \\
CCD0+prSCAN & 1.859 & 887 & 1.783 & 1009 \\
CCD0+trSCAN & 1.853 & 893 & 1.778 & 1016 \\
BD0 & 1.871 & 879 & 1.795 & 999 \\
BD0+pTPSS & 1.849 & 901 & 1.776 & 990 \\
BD0+pSCAN & 1.859 & 884 & 1.784 & 970 \\
BD0+prSCAN & 1.861 & 881 & 1.786 & 1000 \\
BD0+trSCAN & 1.856 & 886 & 1.781 & 1007 \\
CCSD & 1.856 & 891 & 1.791 & 1008 \\
PBE & 1.841 & 896 & 1.776 & 1008 \\
PBEh & 1.828 & 928 & 1.760 & 1052 \\
LC- $\omega$ PBE & 1.821 & 946 & 1.752 & 1080 \\
CASPT2 & 1.863 & 879 & 1.792 & 988 \\
Expt. ${ }^{\text {b }}$ & 1.840 & 895 & $\ldots$ & $\ldots$ \\
\hline \hline
\end{tabular}

${ }^{\mathrm{a}}$ From Refs. 6 and 45, using all-electron basis set.

${ }^{\mathrm{b}}$ From Refs. 6 and $46-48$.
TABLE VI. Bond lengths $\left(\boldsymbol{R}_{\boldsymbol{e}}\right.$ in $\AA$ ) and harmonic vibrational frequencies $\left(\omega_{e}\right.$ in $\left.\mathrm{cm}^{-1}\right)$ for $\mathrm{NoO}$ and $\mathrm{NoO}_{2}$ calculated by various methods. For methods that predict geometries that deviate from linearity in $\mathrm{NoO}_{2}$, the bond angle is shown in parenthesis.

\begin{tabular}{lcccccc}
\hline \hline \multirow{2}{*}{ Method } & \multirow{2}{*}{$\mathrm{NoO}$} & \multicolumn{5}{c}{$\mathrm{NoO}_{2}$} \\
\cline { 2 - 6 } \cline { 4 - 7 } & $R_{e}$ & & $R_{e}$ & $\omega_{\text {as }}$ & $\omega_{\mathrm{s}}$ & $\omega_{\beta}$ \\
\hline BD0 & 2.008 & 1.868 & 703 & 583 & 256 \\
BD0+pTPSS & 1.948 & 1.828 & 665 & 628 & 193 \\
BD0+pSCAN & 1.972 & 1.844 & 636 & 600 & 183 \\
BD0+prSCAN & 1.979 & 1.849 & 666 & 630 & 181 \\
BD0+trSCAN & 1.966 & 1.840 & 682 & 645 & 184 \\
CCSD & 1.923 & 1.832 & 756 & 660 & 173 \\
CCSD(T) & 1.983 & $\ldots$ & $\ldots$ & $\ldots$ & $\ldots$ \\
PBE & 1.911 & $2.045\left(125^{\circ}\right)$ & 523 & 465 & 149 \\
PBEh & 1.903 & $2.036\left(129^{\circ}\right)$ & 516 & 467 & 142 \\
LC- $\omega$ PBE & 1.879 & $1.974\left(131^{\circ}\right)$ & 613 & 542 & 150 \\
\hline \hline
\end{tabular}

large errors in the fitting procedure to obtain the force constants; however, they are consistently estimated to be around $550 \mathrm{~cm}^{-1}$. Likewise, $\mathrm{CCSD}(\mathrm{T})$ data for $\mathrm{NoO}_{2}$ are absent due to the difficulty in converging these calculations. To the best of our knowledge, there are no accurate reference data for these compounds in the literature. Reference 6 reports, based on B3LYP calculations, that the ground states of $\mathrm{NoO}$ and $\mathrm{NoO}_{2}$ are singlet, and we carried out the calculations for singlet states only because of this and the current limitations of applying CCD0 to open shell systems.

The most noticeable feature of Table VI is that BD0, BD0+DFT, and CCSD predict a linear geometry for $\mathrm{NoO}_{2}$, whereas KS-DFT methods yield bent geometries. The bonds in the nobelium compounds are considerably larger than for the $f^{0}$ actinide oxides because the $5 f$ orbitals are completely filled in the former. The effect of dynamic correlation is also much larger: addition of DFT correlation to BD0 leads to decrease in bond lengths of about $0.02-0.05 \AA$, compared to changes the more modest changes (rarely more than 0.02 $\AA)$ observed above. Based on the results for the previous actinide compounds, we could expect BD0+pSCAN and $\mathrm{BD} 0+$ prSCAN to provide accurate estimates for the bond lengths and frequencies for the nobelium oxides. In the case of $\mathrm{NoO}, \mathrm{CCSD}(\mathrm{T}), \mathrm{BD} 0+\mathrm{pSCAN}$, and $\mathrm{BD} 0+$ prSCAN results are highly similar.

\section{CONCLUSIONS AND OUTLOOK}

Singlet-paired coupled cluster and its combinations with DFT can provide accurate estimates for properties of actinide compounds such as geometries and vibrational frequencies. Compared to the data from the most accurate estimates available (CASPT, CCSD(T), experiment), typical deviations of CCD0 and CCD0+DFT methods are around $0.01 \AA$ for bond lengths and $20 \mathrm{~cm}^{-1}$ for harmonic vibrational frequencies. These deviations are similar to the ones computed for simple first- and second-row diatomics in a previous work, ${ }^{13}$ indicating the wide applicability and generality of the approach. The CCD0 and CCD0+DFT results presented here reinforce predictions by other methods 
(e.g., $\operatorname{CCSD}(\mathrm{T})$ or CASSCF) for species for which no experimental data are available (e.g., the important $\mathrm{UO}_{2}^{2+}$ cation), including the instability of the $\mathrm{PuO}_{2}^{4+}$ ion. The BD0 and $\mathrm{BD} 0+\mathrm{DFT}$ results for $\mathrm{NoO}$ and $\mathrm{NoO}_{2}$ are probably the most reliable estimates available so far, as previous reports (see Ref. 6) utilized KS-DFT functionals which, according also to the results here, are not consistently reliable for actinide compounds.

For most of the molecules studied here, $\operatorname{CCSD}(\mathrm{T})$ appears to provide reliable results. CCD0+DFT can provide data of similar quality to $\operatorname{CCSD}(\mathrm{T})$ while being a order in magnitude lower in cost and much more robust for handling static correlation. The CCD0 and CCD0+DFT methods are seen to be more accurate than pCCD for $\mathrm{UO}_{2}^{2+}$. Because pCCD has lower cost than $\mathrm{CCD} 0$ and misses mostly dynamic correlation, the results here suggest that pCCD+DFT combinations analogous to CCD0+DFT (such as those suggested in Refs. 3, 13 , and 49) may be very promising for actinide chemistry. Currently, we are working on some of these pCCD+DFT combinations and on extensions of CCD0 for treating openshells, which would greatly increase the applicability of CCD0-based techniques to actinide chemistry.

\section{ACKNOWLEDGMENTS}

This work was supported by the U.S. Department of Energy, Office of Science, Office of Basic Energy Sciences, Heavy Element Chemistry Program under Award No. DEFG02-04ER15523. G.E.S. is a Welch Foundation Chair (No. C-0036).

${ }^{1}$ P. Pyykkö, J. Li, and N. Runeberg, J. Phys. Chem. 98, 4809 (1994).

${ }^{2}$ C. Heinemann and H. Schwarz, Chem. Eur. J. 1, 7 (1995).

${ }^{3}$ A. J. Garza, T. M. Henderson, I. W. Bulik, and G. E. Scuseria, Phys. Chem. Chem. Phys. 17, 22412 (2015).

${ }^{4}$ I. W. Bulik, T. M. Henderson, and G. E. Scuseria, J. Chem. Theory Comput. 11, 3171 (2015).

${ }^{5}$ D. Wang, W. F. van Gunsterenb, and Z. Chai, Chem. Soc. Rev. 41, 5836 (2012).

${ }^{6}$ A. Kovács, R. J. M. Konings, J. K. Gibson, I. Infante, and L. Gagliardi, Chem. Rev. 115, 1725 (2015).

${ }^{7}$ P. A. Limacher, P. W. Ayers, P. A. Johnson, S. de Baerdemacker, D. van Neck, and P. Bultinck, J. Chem. Theory Comput. 9, 1394 (2013).

${ }^{8}$ P. A. Limacher, T. D. Kim, P. W. Ayers, P. A. Johnson, S. de Baerdemacker, D. van Neck, and P. Bultinck, Mol. Phys. 112, 853 (2014).

${ }^{9}$ P. Tecmer, K. Boguslawski, P. A. Johnson, P. A. Limacher, M. Chan, T. Verstraelen, and P. W. Ayers, J. Phys. Chem. A 118, 9058 (2014).

${ }^{10}$ K. Boguslawski, P. Tecmer, P. W. Ayers, P. Bultinck, S. de Baerdemacker, and D. van Neck, Phys. Rev. B 89, 201106(R) (2014).

${ }^{11}$ T. Stein, T. M. Henderson, and G. E. Scuseria, J. Chem. Phys. 140, 214113 (2014).
${ }^{12}$ T. M. Henderson, I. W. Bulik, T. Stein, and G. E. Scuseria, J. Chem. Phys. 141, 244104 (2014).

${ }^{13}$ A. J. Garza, I. W. Bulik, A. G. Sousa Alencar, J. Sun, J. P. Perdew, and G. E. Scuseria, "Combinations of coupled cluster, density functionals, and the random phase approximation for describing static and dynamic correlation, and van der Waals interactions," Mol. Phys. (to be published).

${ }^{14}$ C. A. Jimenez-Hoyos, T. M. Henderson, and G. E. Scuseria, J. Chem. Theory Comput. 7, 2667 (2011).

${ }^{15}$ P. Tecmer, K. Boguslawski, and P. W. Ayers, Phys. Chem. Chem. Phys. 7, 14427 (2015).

${ }^{16}$ C. E. Dykstra, Chem. Phys. Lett. 45, 466 (1977).

${ }^{17}$ N. C. Handy, J. A. Pople, M. Head-Gordon, K. Raghavachari, and G. W. Trucks, Chem. Phys. Lett. 164, 185 (1989).

${ }^{18}$ R. Kobayashi, N. C. Handy, R. D. Amos, G. W. Trucks, M. J. Frisch, and J. A. Pople, J. Chem. Phys. 95, 6723 (1991).

${ }^{19}$ M. Levy, Proc. Natl. Acad. Sci. U. S. A. 76, 6062 (1979).

${ }^{20}$ E. H. Lieb, Int. J. Quantum Chem. 24, 243 (1983).

${ }^{21}$ H. Stoll, C. E. Pavlidou, and H. Preuss, Theor. Chim. Acta 49, 143 (1978).

${ }^{22}$ C. Lee, W. Yang, and R. G. Parr, Phys. Rev. B 37, 785 (1988).

${ }^{23}$ J. Tao, J. P. Perdew, V. N. Staroverov, and G. E. Scuseria, Phys. Rev. Lett. 91, 146401 (2003).

${ }^{24}$ J. Sun, A. Ruzsinszky, and J. P. Perdew, Phys. Rev. Lett. 115, 036402 (2015).

${ }^{25}$ P. Gori-Giorgi and J. P. Perdew, Phys. Rev. B 69, 041103(R) (2004).

${ }^{26}$ M. J. Frisch, G. W. Trucks, H. B. Schlegel et al., Gaussian, Revision H.21, Gaussian Inc., Wallingford, CT, 2009.

${ }^{27}$ A. Bergner, M. Dolg, W. Kuechle, H. Stoll, and H. Preuss, Mol. Phys. 80, 1431 (1993).

${ }^{28}$ M. Kaupp, P. v. R. Schleyer, H. Stoll, and H. Preuss, J. Chem. Phys. 94, 1360 (1991).

${ }^{29}$ M. Dolg, H. Stoll, H. Preuss, and R. M. Pitzer, J. Phys. Chem. 97, 5852 (1993).

${ }^{30}$ Y.-K. Han and K. Hirao, J. Chem. Phys. 113, 7345 (2000).

${ }^{31}$ E. Fromager, F. Réal, P. Wåhlin, U. Wahlgren, and H. J. Aa Jensen, J. Chem. Phys. 131, 054107 (2009).

${ }^{32}$ H. H. Cornehel, C. Heinemann, J. Marçalo, A. Pires de Matos, and H. Schwarz, Angew. Chem., Int. Ed. Engl. 35, 891 (1996).

${ }^{33}$ V. E. Jackson, R. Cracium, D. A. Dixon, K. A. Peterson, and W. A. de Jong, J. Phys. Chem. A 112, 4095 (2008).

${ }^{34}$ M. Straka, K. G. Dyall, and P. Pyykkö, Theor. Chem. Acc. 106, 393 (2001).

${ }^{35}$ L. Gagliardi and B. O. Roos, Chem. Phys. Lett. 331, 229 (2000).

${ }^{36}$ T. J. Lee and P. R. Taylor, Int. J. Quantum Chem. 36(S23), 199 (1989).

${ }^{37}$ S. Tsushima, J. Phys. Chem. B 112, 13059 (2008).

${ }^{38}$ W. Huang, P. Pyykkö, and J. Li, Inorg. Chem. 54, 8825 (2015).

${ }^{39}$ M. Zhou and L. Andrews, J. Chem. Phys. 111, 11044 (1996).

${ }^{40}$ R. D. Hunt, J. T. Yustein, and L. Andrews, J. Chem. Phys. 98, 6070 (1993).

${ }^{41}$ D. W. Green and G. T. Reedy, J. Chem. Phys. 7, 2921 (1976).

${ }^{42}$ L. Andrews, X. Wang, Y. Gong, G. P. Kushto, B. Vlaisavljevich, and L. Gagliardi, J. Phys. Chem. A 118, 5289 (2014).

${ }^{43}$ P. Pyykkö, S. Riedel, and M. Patzschke, Chem. Eur. J. 11, 3511 (2005).

${ }^{44}$ S. F. Ashley, G. T. Parks, W. J. Nuttall, C. Boxall, and R. W. Grimes, Nature 492, 31 (2012).

${ }^{45}$ A. Kovács and R. J. M. Konings, J. Phys. Chem. A 115, 6646 (2011).

${ }^{46}$ C. T. Dewberry, K. C. Etchison, and S. A. Cooke, Phys. Chem. Chem. Phys. 9, 4895 (2007).

${ }^{47}$ G. Edvinsson and A. Lagerqvist, Phys. Scr. 30, 309 (1984).

${ }^{48}$ G. Edvinsson, L.-E. Selvin, and N. Aslund, Ark. Fys. 30, 28 (1965).

${ }^{49}$ A. J. Garza, T. M. Henderson, I. W. Bulik, and G. E. Scuseria, J. Chem. Phys. 142, 044109 (2015). 